\title{
Endovascular treatment of a ruptured aneurysm arising from the proximal end of a partial vertebrobasilar duplication with a contralateral prominent persistent primitive hypoglossal artery: illustrative case
}

\author{
Nobuyuki Genkai, MD, PhD, ${ }^{1}$ Kouichirou Okamoto, MD, PhD, ${ }^{2}$ Toshiharu Nomura, MD, PhD, ${ }^{1}$ and Hiroshi Abe, MD, PhD ${ }^{1}$ \\ ${ }^{1}$ Department of Neurosurgery, Tachikawa General Hospital, Tachikawa Medical Center, Niigata, Japan; and ${ }^{2}$ Department of Translational Research, Brain Research Institute, \\ Niigata University, Niigata, Japan
}

\begin{abstract}
BACKGROUND Ruptured aneurysms associated with a partial vertebrobasilar duplication or a persistent primitive hypoglossal artery (PPHA) have been reported. Only rarely has endovascular treatment of ruptured aneurysms in association with both vascular variations been reported.

OBSERVATIONS A 66-year-old woman experienced the sudden onset of a severe headache caused by a subarachnoid hemorrhage. Cerebral angiograms demonstrated a prominent PPHA originating from the left internal carotid artery at the C2 vertebral level and a partial vertebrobasilar duplication between the hypoplastic right vertebral artery and proximal basilar artery with a small aneurysm at the proximal end of the duplication from where the anterior spinal artery originated. The left vertebral artery was aplastic. A microcatheter was introduced into the aneurysm via the PPHA under the control of high blood flow, using a balloon-assisted technique. The aneurysm was completely obliterated with a coil. Although small cerebellar and cerebral infarcts developed during the procedure, the patient was discharged without neurological symptoms.
\end{abstract}

LESSONS To avoid serious neurological complications, precise analysis of the complex vascular anatomy, including the anterior spinal artery and hemodynamics, is clinically important for endovascular therapy of cerebral aneurysms in patients with an association between a partial vertebrobasilar duplication and a PPHA

https://thejns.org/doi/abs/10.3171/CASE20108

KEYWORDS partial vertebrobasilar duplication; primitive lateral basilovertebral anastomosis; persistent primitive hypoglossal artery; ruptured aneurysm

Persistent carotid-basilar connections have a low prevalence, and a persistent primitive hypoglossal artery (PPHA) is extremely rare. ${ }^{1}$ Most carotid-basilar connections are incidental findings. However, a high incidence of PPHAs has been associated with cerebral aneurysms. ${ }^{1,2}$ Hypoplasia or aplasia of the vertebral artery (VA) is a common finding in patients with a $\mathrm{PPHA},{ }^{1}$ requiring precise analysis of the vascular anatomy and hemodynamics when treating a ruptured aneurysm in these patients.

The primitive lateral basilovertebral anastomosis (PLBA) is an embryonic, longitudinal, anastomotic channel of the vertebrobasilar system. It can influence the formation of a partially duplicated vertebrobasilar junction with an associated cerebral aneurysm at the junction. ${ }^{3}$

Here, we report the case of a patient with an endovascularly treated ruptured aneurysm arising from the proximal end of the right partial vertebrobasilar duplication, which is the prominent left PPHA-basilar artery (BA) junction, with an aplastic left VA.

\section{Illustrative Case}

A 66-year-old woman who was hyperlipidemic experienced the sudden onset of a severe headache. She was transferred to the

ABBREVIATIONS $3 \mathrm{D}=$ three dimensional; $\mathrm{AICA}=$ anterior inferior cerebellar artery; $\mathrm{ASA}=$ anterior spinal artery; $\mathrm{BA}=$ basilar artery; $\mathrm{CT}=$ computed tomography; $\mathrm{CTA}=$ computed tomography angiography; $\mathrm{DSA}=$ digital subtraction angiography; $\mathrm{ICA}=$ internal carotid artery; $\mathrm{PCoA}=$ posterior communicating artery;

$\mathrm{PHA}=$ primitive hypoglossal artery; PICA = posterior inferior cerebellar artery; PLBA = primitive lateral basilovertebral anastomosis; POA = primitive otic or acoustic artery; $\mathrm{PPHA}=$ persistent primitive hypoglossal artery; $\mathrm{ProA}=$ proatlantal intersegmental artery; $\mathrm{PTA}=$ primitive trigeminal artery; $\mathrm{SAH}=$ subarachnoid hemorrhage; $\mathrm{TCA}=$ trigeminocerebellar artery; $\mathrm{VA}=$ vertebral artery.

INCLUDE WHEN CITING Published May 10, 2021; DOI: 10.3171/CASE20108.

SUBMITTED November 12, 2020. ACCEPTED February 11, 2021.

(C) 2021 The authors, CC BY-NC-ND 4.0 (http://creativecommons.org/licenses/by-nc-nd/4.0/). 


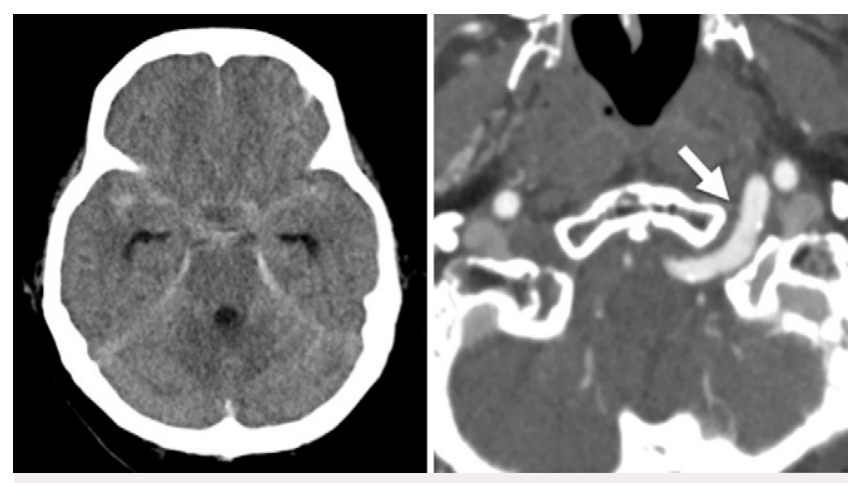

FIG. 1. Left: CT scan of the brain on admission showing an SAH, hyperattenuated basal cisterns. Right: A source image of 3D-CTA demonstrating the left PPHA (arrow) entering into the skull through the left hypoglossal canal.

emergency department of Tachikawa General Hospital. Brain computed tomography (CT) revealed a subarachnoid hemorrhage (SAH) (Fig. 1 left). Subsequent three-dimensional (3D)-CT angiography (CTA) demonstrated a prominent left PPHA, which originated from the left internal carotid artery (ICA) at the C2 vertebral level and entered the skull through the left hypoglossal canal (Fig. 1 Right, arrow). A small cerebral aneurysm was also demonstrated in the anterior wall of the PPHA-BA junction, with an aplastic left VA. Digital subtraction angiography (DSA) and 3D-rotational angiography confirmed the presence of the left PPHA (Fig. 2A-C, arrows and Fig. 2D) and the aneurysm $(2.8 \mathrm{~mm} \times 2.7 \mathrm{~mm} \times 2.4 \mathrm{~mm})$. In addition, cerebral angiograms demonstrated a right vertebrobasilar anastomosis between the hypoplastic right VA and the proximal BA, and the left trigeminocerebellar artery (TCA) originating from the distal portion of the BA (Fig. 2C, paired arrowheads, and Fig. 2D). The anterior spinal artery (ASA) originated from the posterior aspect of the left PPHA-BA junction (Fig. 2C, small arrows, and Fig. $2 \mathrm{E}$, arrows), which is the proximal end of the right vertebrobasilar anastomosis, with the partial remnant right PLBA between the hypoplastic right $\mathrm{VA}$ and the right anterior inferior cerebellar artery (AICA) as a partial vertebrobasilar duplication (Fig. 2D). The common trunk of the left AICA-posterior inferior cerebellar artery (PICA) originated from the BA at the level of the distal end of the duplication (Fig. 2D). The left posterior communicating artery (PCOA) was not demonstrated.

The aneurysm was small and had a broad neck; therefore, treatment via stent-assisted coiling was initially planned. The patient was administered general anesthesia and heparin. A 90-cm 6-Fr Roadmaster TH2 catheter (Goodman) was introduced into the left PPA through the left ICA. However, high blood flow through the PPHA hampered the stent-assisted technique. A balloon-assisted technique was then attempted, using an inflated balloon catheter (Sceptor C, $4.0 \mathrm{~mm} \times 10 \mathrm{~mm}$, Terumo). An Excersior SL-10 catheter (Stryker) was then guided into the aneurysm with a CHIKAl-14 black soft tip guidewire (Asahi Intech), and the aneurysm was successfully embolized through the catheter with a $2.0-\mathrm{mm} \times$ 2.0-mm $\times 6.0-\mathrm{cm}$ Axium Prime HELICAL coil (eV3 Covidien). Complete obliteration of the aneurysm was confirmed on the DSA performed immediately after the procedure.
Antiplatelet therapy was started the next morning with clopidogrel, $75 \mathrm{mg} /$ day, and cilostazol, $200 \mathrm{mg} /$ day. A CT scan obtained the next day showed small infarcts in the left cerebellar hemisphere, in the distribution of the left AICA-PICA, and the left occipital lobe. Review of the DSA performed immediately after the procedure confirmed occlusion of the common trunk of the left AICA-PICA (Fig. 3, paired arrows). The patient exhibited no neurological signs or symptoms and was discharged after she achieved a modified Rankin Scale score of 0 . At discharge, fluid-attenuated inversion recovery images from magnetic resonance imaging of the brain identified these small infarcts (Fig. 4).

\section{Discussion \\ Observations}

Our patient developed a ruptured aneurysm from the anterior wall of the proximal end of the right partial vertebrobasilar duplication, where the left PPHA-BA junction was located. In the duplication, a persistent segment of the right-side PLBA connected the hypoplastic right VA and right AICA. The left VA was aplastic, and the ASA originated from the posterior aspect of the proximal end of the duplication. In order to avoid serious neurological complications, precise analysis of the complex vascular anatomy, including the ASA and hemodynamics, is essential in endovascular therapy for patients with various posterior circulation. Ruptured aneurysms associated with a partial vertebrobasilar duplication or a PPHA have been reported; however, case reports focusing on the origin of the ASA and endovascular treatment of ruptured aneurysms in association with both vascular variations are extremely limited.

\section{Lessons}

The primitive trigeminal artery (PTA), primitive otic or acoustic artery (POA), primitive hypoglossal artery (PHA), and proatlantal intersegmental artery (ProA) are persistent fetal anastomoses existing between the carotid and vertebrobasilar arteries. ${ }^{1,4-6}$ These emerge at the 4- to 5-mm embryonic stage and regress roughly at the rate at which the PCoAs and VAs develop. The rarest, and the first to disappear, is the POA, followed by the PHA, PTA, and ProA. ${ }^{6}$ Occasionally, the persistence of these vessels is noted after birth and into adult life. A PPHA is one of these rare embryological remnants, and hypoplasia or aplastic VAs are common in patients with a PPHA. ${ }^{1}$ Proposed criteria for the anatomical and angiographic definitions of a PPHA are as follows: 1) the artery arises from the ICA at the C1-3 level, 2) the PPHA enters the skull via the hypoglossal canal, 3) the BA is filled only beyond the point of entry of the PPHA, and 4) there is no PCoA. ${ }^{4}$ Our patient satisfied all four criteria. Despite a low angiographic incidence $(0.05 \%),{ }^{2}$ cases of more than 160 patients with a PPHA have been reported. ${ }^{6}$ Our patient developed a ruptured aneurysm from the anterior wall of the left prominent PPHA-BA junction. The left VA was aplastic, and the ASA originated from the posterior aspect of the junction.

The common trunk of the left AICA-PICA of the patient in this report originated from the $B A$ at the level of the distal end of the partial vertebrobasilar duplication. In addition, the left TCA branched off from the distal portion of the BA and perfused the part of the cerebellum that is normally supplied by the AICA. The course of the TCA was confirmed by source images of 3D-CTA in contact with the root of the left trigeminal nerve. The TCA has rarely been described in reports, and it has not been well recognized as a cerebellar artery. ${ }^{7,8}$ The TCA may be regarded as an AICA with a high 


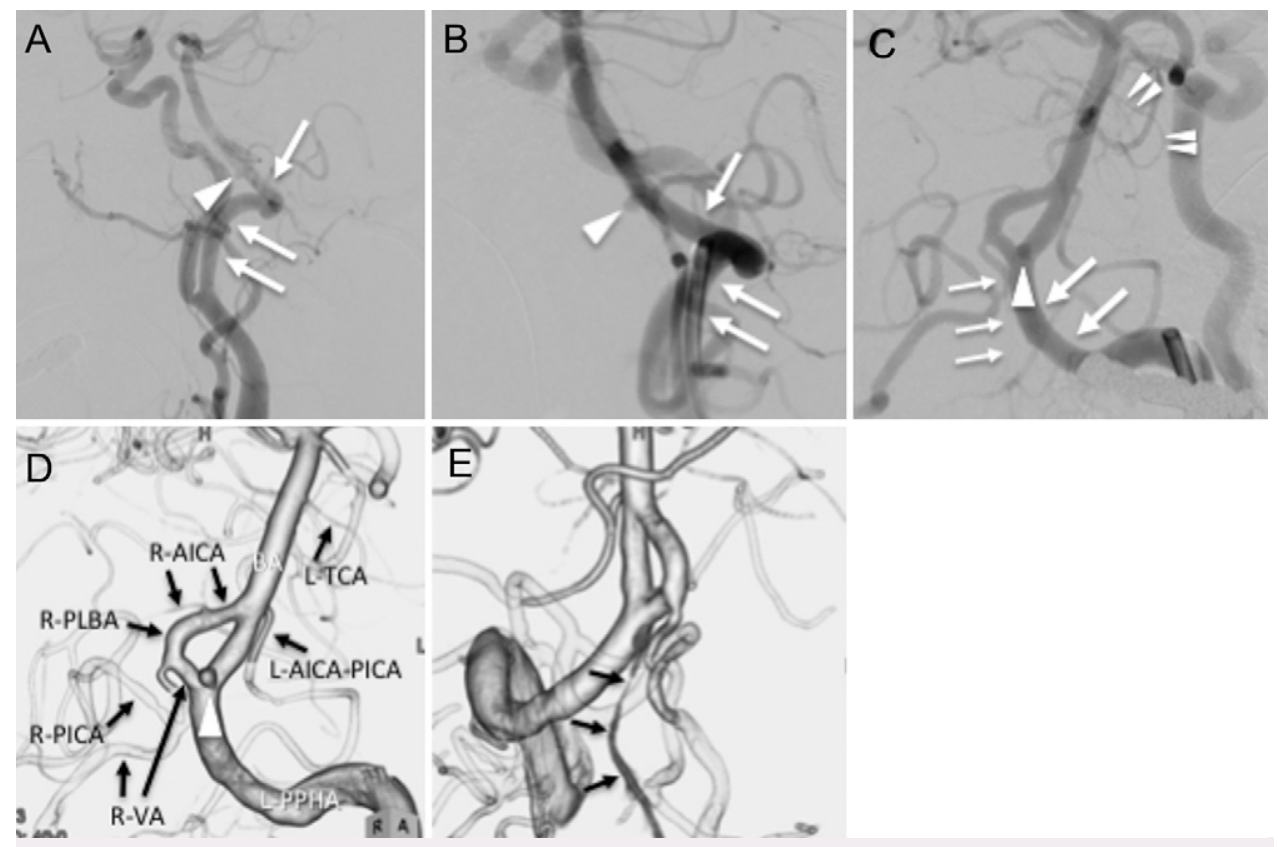

FIG. 2. A: Lateral view of the left carotid artery angiogram (DSA) demonstrating the PPHA originating from the left ICA at the $\mathrm{C} 2$ vertebral level (arrows). The PCOA is not visible. A small aneurysm arises from the anterior wall of the junction of the left PPHA and the BA (arrowhead). B: Lateral view of the selective PPHA (arrows) angiogram (DSA) showing the aneurysm (arrowhead) at the junction. Dimensions of the aneurysm are $2.8 \mathrm{~mm}$ $\times 2.7 \mathrm{~mm} \times 2.4 \mathrm{~mm}$. C: Anteroposterior view of the selective PPHA angiogram (DSA) demonstrating the aneurysm (large arrowhead) at the junction of the left PPHA (large arrows) and the BA, which is the proximal end of a partial vertebrobasilar duplication, and the left TCA originating from the distal BA (double arrowheads). The ASA originates from the junction of the left PPHA and BA (small arrows). D: Anterior view of the selective PPHA angiogram (3D-rotation angiogram). The aneurysm (arrowhead) is located in the anterior wall of the proximal end of the partial vertebrobasilar anastomosis composed of the right (R)-VA, the R-AICA, and a partially persistent segment of the R-PLBA between them, as the right partial vertebrobasilar duplication. The proximal end of the duplication is the junction of the left (L)-PPHA and the BA. The common trunk of the left (L)-AICAPICA originates from the BA at the level of the distal end of the duplication. The L-TCA originates from the distal segment of the BA. E: Magnified posterior view of the selective PPHA angiogram (3D-rotation angiogram) showing the ASA (black arrows) originating from the posterior aspect of the proximal end of the duplication, which is the junction of the left PPHA and the BA.

origin. If only the rostral segment of the PTA regresses, the distal segment persists and develops as the TCA, representing a caudal remnant of the PTA. ${ }^{7,8}$ Coexistence of different persistent primitive arteries on the same side is extremely rare, ${ }^{9,10}$ and association of the TCA with a PPHA or other persistent primitive arteries has not been reported.

The incidence of cerebral aneurysms associated with a persistent PTA is approximately $4 \%$, similar to that of the general population; ${ }^{11}$ however, the incidence that is associated with a PPHA is much higher $(26.9 \%-27.6 \%))^{1,2}$ Of these aneurysms, $31.4 \%$ are located at the PPHA-BA junction and $53 \%$ are found in the posterior circulation. ${ }^{4}$ Hemodynamic stress and/or inherent fragility of the vascular wall may play a role in ruptured aneurysms associated with persistent carotidbasilar connections, including a PPHA. ${ }^{1,4}$ In an autopsy case of SAH associated with multiple cerebral aneurysms in the anterior circulation and coexisting with the left PPHA, aplastic left VA, and hypoplastic right $\mathrm{VA}$, a microscopic examination revealed atherosclerotic changes in the PPHA and true aneurysmal changes in multiple aneurysms, with defects in the tunica media at all arterial bifurcations, without early aneurysmal changes. ${ }^{12}$
Gregg suggested that some variations in the vertebrobasilar circulation might be associated with a partially persistent PLBA. ${ }^{3}$ The PLBA can participate in the formation of a partially duplicated vertebrobasilar junction, a variant that needs to be distinguished from a basilar fenestration. ${ }^{3}$ The latter results either from the nonfusion of the ascending rami of the dorsal branch of the ProA (proximal fenestration) or from the partial or complete nonfusion of the paired longitudinal neural arteries (distal fenestration) in the 7- to 12-mmlong embryo when the embryonal carotid-basilar connections begin to regress and disappear. ${ }^{3,4}$ Conversely, in a vertebrobasilar duplication, the lateral limb is made of a persistent segment of the PLBA, whereas the medial limb corresponds to the normal vertebrobasilar junction. ${ }^{3}$ In our patient, the normal vertebrobasilar junction was replaced by the prominent PPHA-BA junction.

$B A$ fenestrations are most frequently located in the proximal $B A$ trunk, close to the junction of the VAs $(73 \%)$, and $35.5 \%$ have been associated with vertebrobasilar junction aneurysms. Histopathological examinations of intracranial fenestrations have demonstrated structural defects in the media of the medial walls at the two ends of the 


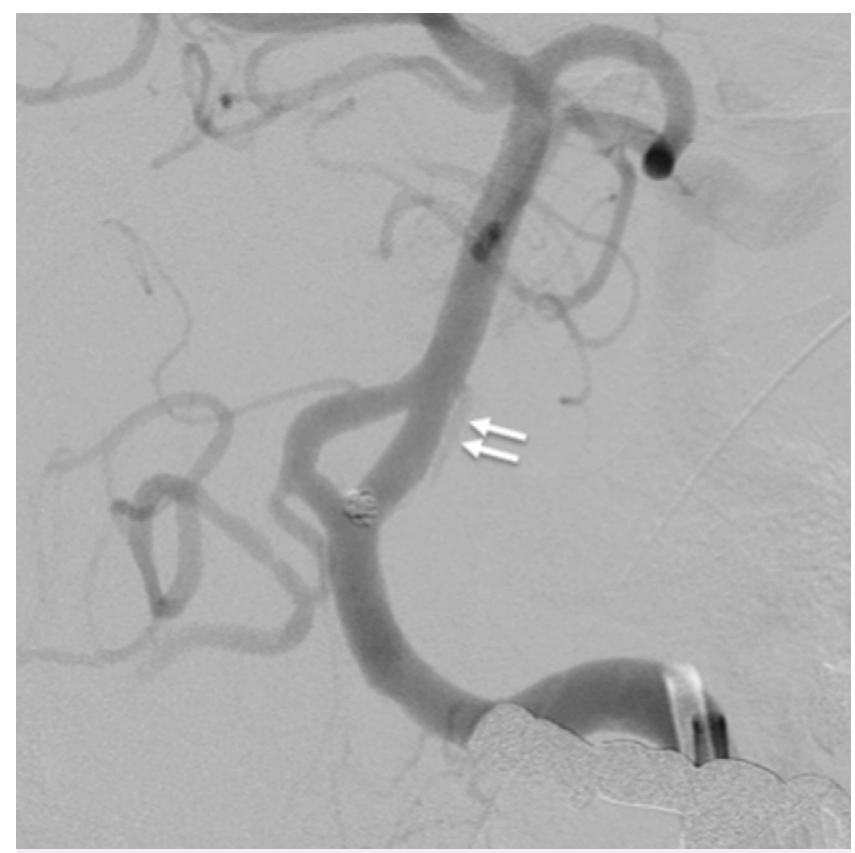

FIG. 3. Anteroposterior view of the selective PPHA artery angiogram (DSA) after coil embolization showing a coil successfully introduced into the aneurysm and obstruction of the common trunk of the L-AICA-PICA (double arrows).

fenestration. These muscular gaps and increased hemodynamic stresses have been suggested as factors involved in the high incidence of aneurysms at the proximal end. ${ }^{3}$ In this patient, partial vertebrobasilar duplication was formed between the distal right VA and proximal BA. The ruptured aneurysm developed from the anterior wall of the proximal end of the duplication. A similar cerebral aneurysm arising from the proximal end of a partial vertebrobasilar duplication without a PPHA has been documented in the literature. ${ }^{3}$ In our patient, the considerably complex hemodynamics associated with partial vertebrobasilar duplication and the prominent PPHA seemed to contribute to the development and rupture of the aneurysm at the proximal end of the duplication, where the left PPHA-BA junction is, and to the occurrence of asymptomatic ischemic complications in the posterior circulation, including the left AICA-PICA distribution.

Recently, endovascular therapies have reportedly been performed for cerebral aneurysms associated with the PPHA and BA fenestration ${ }^{4}$ and for those associated with the fenestration or duplication of the VA. ${ }^{13}$ In cases of prominent PPHA with bilateral hypoplastic and/or aplastic VAs, as described here, control of the high blood flow through the PPHA is imperative for successful endovascular therapy. In the patient described in this report, the insertion of a microcatheter into the cerebral aneurysm was difficult without the balloon-assisted technique because of high blood flow. Although the technique had a risk for ischemic complication in the posterior circulation by obstructing the high blood flow to the BA through the PPHA, coil embolization was successfully achieved in a short time without serious complications using this technique.

In most cases, the ASA is formed by the fusion of the left and right trunks originating from the VAs. ${ }^{14,15}$ The arteries in the posterior

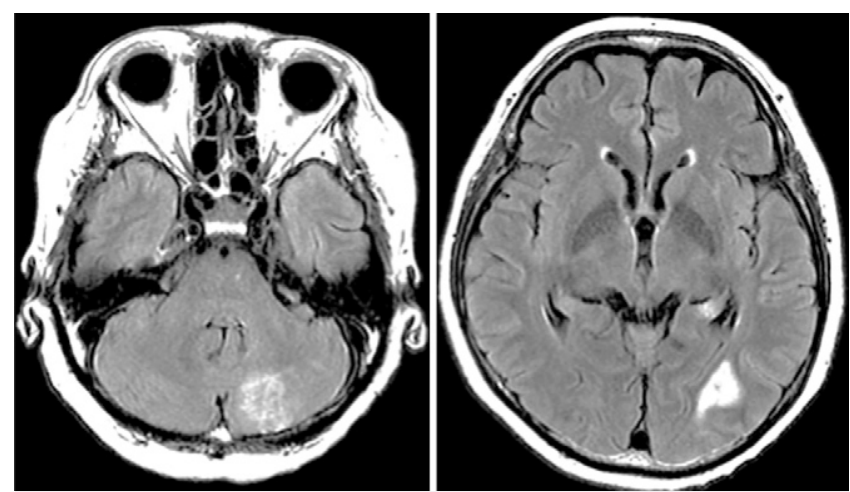

FIG. 4. Fluid-attenuated inversion recovery images of the brain at discharge showing small infarcts in the left cerebellar hemisphere in the L-AICA-PICA distribution and the left occipital lobe.

circulation show greater anatomical variations than cerebral arteries in other locations. The ASA is no exception, and it originates as a single trunk in some cases. ${ }^{14,15}$ In the case of occlusion of the VA where there is bilateral participation of the main ASA trunk, the medulla oblongata might still receive adequate blood supply from the opposite ramus via collateral branches. Unfortunately, with endovascular therapy, devastating complications can occur. These are often related to direct obliteration of the ASA at its origin or to secondary thrombus extension involving the ASA origin, ${ }^{14}$ in particular with a single trunk, as in the case described in this report. If the aneurysm were larger and its neck wide, a flow division technique would be used as an alternative endovascular treatment even though it has inherent risks, such as occlusion of the single trunk of the ASA as a side branch of the prominent PPHA. ${ }^{16}$ The origin of the ASA has not been investigated in patients with a PPHA. The left VA was aplastic and the right VA was hypoplastic in our patient. The PPHA-BA junction was the proximal end of the partial vertebrobasilar duplication where the right VA was connected to the junction from which the ASA originated. Therefore, the ASA could receive blood flow through the right VA during the balloon-assisted procedure.

In particular, endovascular iatrogenic occlusion of the origin of the ASA, or its ramus, is commonly employed as therapy for aneurysms of the vertebrobasilar system. Knowledge of anatomical variations, including the origin and course of the ASA, as presented here, has critical clinical importance for surgery and endovascular procedures involving this area.

\section{References}

1. Varvari I, Bos EM, Dinkelaar W, et al. Fatal subarachnoid hemorrhage from an aneurysm of a persistent primitive hypoglossal artery: case series and literature overview. World Neurosurg. 2018;117:285-291.

2. Yamanaka K, Noguchi K, Hayasaki K, Matsuoka Y. Persistent primitive hypoglossal artery associated with arteriovenous malformation—case report. Neurol Med Chir (Tokyo). 1990;30(12):949-955.

3. Gregg L, Gailloud P. The role of the primitive lateral basilovertebral anastomosis of Padget in variations of the vertebrobasilar arterial system. Anat Rec (Hoboken). 2017;300(11):2025-2038.

4. Kanematsu M, Satoh K, Nakajima N, et al. Ruptured aneurysm arising from a basilar artery fenestration and associated with a persistent primitive hypoglossal artery. Case report and review of the literature. J Neurosurg. 2004;101(3):532-535. 
5. Garza-Mercado R, Cavazos E. Persistent trigeminal artery associated with intracranial arterial aneurysm. Neurosurgery. 1984;14(5): 604-607.

6. Luh GY, Dean BL, Tomsick TA, Wallace RC. The persistent fetal carotid-vertebrobasilar anastomoses. AJR Am J Roentgenol. 1999; 172(5):1427-1432.

7. Marinković S, Gibo H, Nikodijević I. Trigeminocerebellar arteryanatomy and possible clinical significance. Neurol Med Chir (Tokyo). 1996;36(4):215-219.

8. Amagasaki K, Abe S, Watanabe S, et al. Trigeminal neuralgia caused by a trigeminocerebellar artery. J Neurosurg. 2014;121(4):940-943.

9. Uchino A, Saito N, Inoue K. Type 2 proatlantal intersegmental artery associated with persistent trigeminal artery diagnosed by MR angiography. Surg Radiol Anat. 2012;34(8):773-776.

10. Hayashi K, Hashiguchi A, Moroki K, et al. Co-existence of persistent primitive trigeminal artery and another primitive carotid-basilar anastomosis associated with segmental agenesis of the ipsilateral internal carotid artery: a case report. Egypt J Neurosurg. 2019;33:1.

11. Murai S, Sugiu K, Hishikawa T, et al. Endovascular treatment for unruptured aneurysm associated with persistent primitive trigeminal artery: a case report and literature review. Acta Neurochir (Wien). 2019;161(2):407-411.

12. Fujii $Y$, Arai $H$, Takeuchi S, et al. An autopsy case of persistent primitive hypoglossal artery with multiple cerebral aneurysms. Article in Japanese. No Shinkei Geka. 1988;16(4):421-426.

13. Okamura A, Kawamoto $Y$, Sakoda E, et al. A case of coil-embolized unruptured saccular aneurysm associated with fenestration of the intracranial vertebral artery. Article in Japanese. JNET J Neuroendovasc Ther. 2013;7(4):275-280.

14. Er U, Fraser K, Lanzino $\mathrm{G}$. The anterior spinal artery origin: a microanatomical study. Spinal Cord. 2008;46(1):45-49.

15. Songur A, Gonul $Y$, Ozen $O A$, et al. Variations in the intracranial vertebrobasilar system. Surg Radiol Anat. 2008;30 (3):257-264.

16. Dandapat S, Mendez-Ruiz A, Martínez-Galdámez M, et al. Review of current intracranial aneurysm flow diversion technology and clinical use. J Neurointerv Surg. 2021;13(1):54-62.

\section{Disclosures}

The authors report no conflict of interest concerning the materials or methods used in this study or the findings specified in this paper.

\section{Author Contributions}

Conception and design: Genkai, Okamoto, Abe. Acquisition of data: Genkai, Nomura, Abe. Analysis and interpretation of data: Okamoto, Abe. Drafting the article: Genkai, Okamoto, Abe. Critically revising the article: Genkai, Okamoto, Abe. Reviewed submitted version of manuscript: Okamoto, Abe. Statistical analysis: Abe. Administrative/technical/material support: Abe. Study supervision: Okamoto, Abe.

\section{Correspondence}

Nobuyuki Genkai: Niigata Neurosurgical Hospital, Niigata, Japan. genkai4926@yahoo.co.jp. 